\title{
Les stances devant la mort dans le théâtre baroque ou le Retrait du
} Sage

Claude Reichler

\section{Citer ce document / Cite this document :}

Reichler Claude. Les stances devant la mort dans le théâtre baroque ou le Retrait du Sage. In: Littérature, ${ }^{\circ} 75,1989$. La voix, le retrait, l'autre. pp. 79-91;

doi : 10.3406/litt.1989.1502

http://www.persee.fr/doc/litt_0047-4800_1989_num_75_3_1502

Document généré le 01/06/2016 


\section{LES STANCES DEVANT LA MORT DANS LE THÉĀTRE BAROQUE . OU LE RETRAIT DU SAGE *}

Le problème qu'on pose ici est celui du lien entre une forme littéraire - les stances - et le contexte historique et social. On ne cherchera nullement dans les textes des indices documentaires permettant de décrire une réalité historique "positive"; on ne tentera pas non plus d'y découvrir le reflet, plus ou moins déformé, d'enjeux sociaux ou politiques. L'une et l'autre de ces démarches, souvent pratiquées en histoire ou en histoire littéraire, réduisent les textes soit à une fonction de traces ou d'archives, soit à un statut d'expression prédéterminée. Je voudrais montrer la nature symbolique du lien entre une forme littéraire et son contexte : le texte littéraire, d'une certaine façon, donne au monde une intelligibilité en le représentant, puisque le réel n'est que confusion et violence avant qu'il ne soit interprété dans des modèles configurants; mais ceux-ci ne naissent pas dans les textes, qui sont eux-mêmes configurés, notamment par les traditions, par le langage ou par l'histoire. Les textes sont donc historiques de plusieurs façons : parce qu'ils sont modélisés par les transformations qui affectent les sociétés; parce qu'ils donnent à connaître, de façon spécifique et irremplaçable, les modélisations elles-mêmes; et parce qu'ils deviennent, pour les lecteurs ultérieurs, les porteurs d'une historicite que ceux-ci construisent différentiellement, mais qu'ils pourront aussi intégrer dans leur présent à un niveau de synthèse supérieur.

\section{La question des stances}

Les stances dans la tragédie française ont fait l'objet de deux recherches relativement récentes : l'une de Jacques Scherer, dans son grand livre sur le théâtre classique, et l'autre de Jacques Morel '. Ces deux études apportent tous les éléments nécessaires à l'approche d'un problème fort intéressant et trop peu travaillé. Elles recensent un grand nombre de pièces qui contiennent des stances, apparues durant un laps de temps précisément délimité (de 1634 à 1664), au cours de ce demi-siècle

\footnotetext{
* Je voudrais remercier Roger Chartier et Christian Jouhaud, qui m'ont invité à présenter une première version de cette étude dans le cadre de leurs recherches sur les rapports entre histoire et littérature, à l'Ecolc pratique des hautes études en sciences sociales. D’autre part, les réflexions qui suivent trouvent leur appui spéculatif dans l'essai intitulé "La littérature comme interprétation symbolique " in L'interprétation des textes, sous la dir. de C. Reichler, Minuit, 1989.

1. Jacques Scherer, La Dramaturgie classique en France, Paris, [1950], pp. 285-297. Jacques Morel, "Les stances dans la tragédie française au XVII siecle ", in $X V I I^{\circ}$ siécle, Paris, 1965, $\mathrm{n}^{\circ} 66-67, \mathrm{pp} .43-56$.
} 
où certains historiens ont voulu voir les balbutiements du classicisme et où d'autres ont fait apparaître, tout à l'opposé, une esthétique baroque. Elles traitent, de manière exhaustive, de l'origine des stances, de leurs particularités métriques, de leur insertion dans les pièces, de leurs valeurs expressives. L'étude de J. Morel s'efforce de dégager leurs fonctions à l'aide des remarques contenues chez les théoriciens du théâtre. On voit bien, dans cet article très érudit, l'hypothèque que fait peser sur la compréhension du phénomène la nécessité de répondre d'une "évolution " du théâtre français vers le triomphe du classicisme. Mais on sait que cette mise en perspective relève elle-même d'une interprétation, historique et "intéressée ", même et surtout si son premier garant fut Boileau. Elle impose un ordre, elle dicte des problèmes, elle en masque d'autres, elle oriente les solutions.

Je proposerai quant à moi de construire la question des stances à partir d'un emploi significatif dans un texte précis, et de tenir cet emploi pour une compréhension pratique, élaborée à même l'œuvre, d'un aspect des stances, de leurs fonctions et de leurs valeurs historiques et esthétiques. Au contraire des deux études précitées, je n'ambitionnerai pas de parler de toutes les stances, ou des stances en général, mais de certaines seulement, à partir desquelles il paraît possible de délimiter une sorte de «noyau conceptuel», une approximation de quelque chose d'essentiel qu'elles auraient eu charge de mettre en forme dans leur brève histoire, et à quoi elles auraient été admirablement adéquates.

\section{La Mort de Sénèque}

Tristan L'Hermite écrivit La Mort de Sénèque en 1644 pour L'Illustre Théâtre ; ce fut la première pièce montée par Jean-Baptiste Poquelin et ses associés. On peut la rattacher à un ensemble de textes divers qui portent sur Néron et les épisodes de son règne, et qui alimentent la réflexion indirecte que le $\mathrm{XVII}^{\mathrm{e}}$ siècle a menée sur le pouvoir et le mal politique. L'histoire est connue : c'est la conjuration de Pison, découverte par Néron et noyée dans le sang. Néron profite de la circonstance pour se débarrasser de Sénèque, son ancien précepteur, de qui il a appris les principes du gouvernement et ceux de la vertu, mais aussi l'art de la parole, oratoire et poétique. Tristan suit à peu près le récit de Tacite. Il faut noter pourtant qu'il rend Sénèque plus étranger au complot que ne l'avait fait Tacite.

L'argument de la pièce est parfaitement rassemblé et clair. A la première scène, Néron et Sabine se réjouissent d'avoir évincé tous leurs ennemis. Sabine jette cependant sur Sénèque de graves soupçons. A la scène suivante, Sénèque apparaît : il vient demander à Néron la permission de lui rendre les biens qu'il a reçus de lui et de se retirer loin de la cour. Néron refuse. L'acte II est rempli des rencontres et des discours des conjurés. Sénèque, pressenti, refuse de se joindre à eux. Les actes III et IV sont consacrés aux enquêtes et interrogatoires menés par Néron sur dénonciations, aux confrontations et à la découverte du complot. Au dernier acte, Sénèque reparaît ; il occupe le centre de l'acte, d'abord par les stances qu'il prononce à la première scène, ensuite par une longue discussion sur la mort avec sa femme Pauline, enfin par le récit de son suicide et de ses dernières paroles, effectué devant Néron par le centenier qui était chargé d'apporter l'ordre de mort. 
Ce résumé ne dit rien pourtant de l'intérêt central de la pièce, à savoir du problème de la parole comme ressort des actions et comme action elle-même. Dans La Critique de l'École des femmes, Molière explique toute l'importance que prend dans sa pièce l'action de la parole, l'effet produit par les récits sur les personnages. Dans La Mort de Sénèque. Tristan exhibe lui aussi les menées de la parole et ses pièges, mais dans un contexte politique : c'est la calomnie, la délation, l'interrogation; c'est l'exhortation, la persuasion, la simulation... Toutes les facettes de la rhétorique sont là, mais aussi le problème du silence et de l'aveu, et celui de la parole feinte et de la parole vraie.

\section{Deux personnages emblématiques}

Dc̀s l'cntrée, Sabine jette le discrédit sur Sénèque et le présente comme le dernier obstacle à la toute-puissance de Néron, le dernier souvenir de son asservissement. Sénèque est riche des libéralités du prince, et il se fait le contempteur des riches dans ses écrits ; il entretient autour de lui une clientèle d'affranchis et de flatteurs, pendant qu'il méprise la cour; il se donne pour un sage alors qu'il n'est qu'un " pédant hypocrite ". Mais surtout, Sabine fait passer Sénèque pour un redoutable manipulateur du langage, un fat éloquent, habile à surprendre autrui par la ruse de ses arguments et la perfidie de ses insinuations.

Le portrait de Sénèque composé par Sabine contient deux traits essentiels, qui valent l'un pour l'autre, la parole duplice et l'argent. Il n'est pas tant à l'image de Sénèque, qu'à celle des préoccupations du pouvoir. Sabine accuse Sénèque de se parer des attributs de Néron, de sa richesse et de son art d'acteur et d'orateur, et d'usurper ainsi deux fonctions, celle de donner et celle de dire, qui devraient être l'apanage du seul souverain.

Il y a dans le théâtre baroque un perpétuel échange entre le littéral et le figuré, une sorte de permanente symbolisation des objets, des actions, des personnages.Le poignard de Pyrame rend visible la violence impuissante qui se tourne contre le sujet lui-même : il est symbole d'auto-accusation. La robe de Médée, empoisonnée par magie, brûle la jeune femme qui s'en est emparée : c'est qu'elle réalise maléfiquement le feu du désir et l'ardeur des sens portée vers un objet interdit. On accuse Marianne de vouloir empoisonner Hérode; c'est pure calomnie : mais le poison prétendu manifeste la violence de sa parole et la force de sa haine. Genest joue le chrétien converti ; et le jeu devient réalité, le simulacre devient la chose, la représentation ou la figure deviennent ce à quoi elles sont substituées ${ }^{2}$. Il en est ainsi pour Sénèque et Néron : ce sont des emblèmes, l'un du Pouvoir, du politique, l'autre du Savoir, du philosophe, - en lutte pour l'appropriation de la parole. Nul doute que le théâtre baroque n'élabore ici une interprétation symbolique d'enjeux qui ont leur lieu dans la réalité sociale. Il ne s'agit nullement de découvrir une « application " politique, mais

2. C'est tout cela que ne comprend plus Boileau, lorsqu'il se moque de l'outrance des figures baroques ; elles sont pour lui pur décor, et d'un goût douteux. Cette méconnaissance de la valeur symbolique constitue une plus profonde divergence que la querelle des règles et des unités. 
le dessin d'une modélisation historique qui touche à l'exercice de la pensée et de la parole dans leur rapport à l'espace politique. De plus, la figure de Sénèque n'est pas seulement celle du philosophe et du sage ; elle est aussi celle de l'homme de théâtre dont l'œuvre est très connue et admirée à la fin $d u X X V^{e}$ et au début du XVII siècle. On sait d'autre part les prétentions de Néron à la scène et aux succès d'acteur. L'affrontement de ces deux personnages, l'échange entre le politique et le dramatique, offrent une figuration d'un débat qui intéresse également le théâtre et son rôle dans le jeu des représentations sociales.

\section{Rhétorique et politique}

L'entrée de Sénèque, à la scène II, donne un démenti immédiat aux accusations de Sabine. Sénèque demande à l'empereur la permission de se dépouiller de tout, de quitter sa charge et de s'éloigner dans une retraite. La tirade de Sénèque est fort longue, tout comme la réponse de Néron : ce sont deux morceaux de rhétorique parfaitement composés. L'élève prétend battre le maître sur son terrain et argumente, dans un discours où les topoï trop préparés se succèdent les uns aux autres et où le miel des compliments parvient mal à faire oublier la force brute et la méfiance du despote. La conclusion, qui tombe finalement, est plus en accord avec les mobiles dissimulés qu'avec les raisonnements déployés :

Ne me parle donc plus de cet éloignement, Et demeure toujours en ton appartement ${ }^{3}$.

Néron est devenu le maître de la parole, le maître du maître. Mais cette appropriation indue, captation dans le sens négatif - et fort peu rhétorique - du terme, n'est pas le seul aspect de la parole politique. Les scènes de la conjuration constituent de très belles variations sur le thème du langage. A l'acte II, le long discours d'Épicaris, lui aussi parfaitement composé, est un admirable exemple d'éloquence "révolutionnaire". Sabine, elle, règne sur le royaume incertain de l'espionnage et de la délation, de la complicité et de l'indiscrétion. Elle prospère dans ce terrain instable où les fausses confidences et le mensonge lient la parole à la conception machiavélienne du pouvoir ${ }^{4}$. Les longues scènes consacrées à l'enquête de Néron exposent à satiété les mécanismes du secret (qu'il faut savoir garder sans pourtant se taire) et de l'aveu qu'il faut obtenir tout en sachant déjà (puisque le soupçon est un savoir partiel et incertain). Ne manquent pas non plus la violence extrême de la question, ni les variations sur la simulation. Tous les personnages simulent: Néron la clémence, les accusés l'innocence, les délateurs le zèle, et jusqu'aux militaires, qui simulent leur propre naïveté.

3. Je cite d'après l'édition de la Pléiade établie par J. Scherer (et J. Truchet pour le t. II) : ici Théâtre du XVII siècle. t. II, 1986, p. 342, v. 307-308.

4. Voici la «bonne maxime » qu’elle suggère à Néron :

"Pour s'assurer d'un trône, il faut être capable

De confondre parfois innocent et coupable " (v. 37-38). 
Il est impossible de rendre transparente la parole politique. Elle ne fonctionne que dans le secret, dans cette logique qui divise le corps social et exige la reddition des savoirs privés; elle se nourrit de la simulation et de la perfidie qu'elle impose.

\section{Une parole vraie}

Dans cet espace où tout discours est entaché de corruption ou de violence, une seule parole est à la fois conforme à la vérité et à la mesure : celle de Sénèque. Cette parole a son chant, où elle se symbolise, comme le veut le théâtre baroque. Ce sont les stances de l'acte $\mathrm{V}$, scène I :

Mon âme, apprête-toi pour sortir tout entière

De cette fragile matière

Dont le confus mélange est un voile à tes yeux :

Tu dois te réjouir du coup qui te menace,

Pensant te faire injure on va te faire grâce ;

Si l'on te bannit de ces lieux

En t'envoyant là-haut, c'est chez toi qu'on te chasse,

Ton origine vient des Cieux.

Nous avons assez vu le cours de la Nature,

Sa riche et superbe structure,

Ses divers ornements et ses charmants attraits;

Elle a peu de beautés qui ne nous soient connues,

Il faut quitter la terre, et monter sur les nues,

Pour connaître d'autres secrets ;

Il faut chercher du Ciel les belles avenues,

Et voir le Soleil de plus près.

On ne trouve ici-bas que des lois tyranniques,

D'où naissent des effets tragiques,

Et les Monstres y sont au-dessus des Héros;

La Vertu sous le joug y demeure asservie :

L'Orgueil, l'Ambition, l'Avarice et l'Envie

Nous y troublent à tout propos:

Mais là-haut dans l'état d'une meilleure vie

On goûte un éternel repos.

Principe de tout être où mon espoir se fonde,

Esprit qui remplis tout le monde,

Et de tant de bontés favorises les tiens,

Tu vois les cruautés de qui je suis la proie,

Et j'attends de toi seul mon repos et ma joie ;

Fais que je goûte de tes Biens,

Et me tire bientôt, afin que je te voie,

$\mathrm{Du}$ joug de ces pesants liens.

Les stances constituent le monologue d'un personnage retiré du monde des actions, et dont le discours échappe aux contraintes de l'échange, à la persuasion comme à la simulation. S'il diverge de l'alexandrin convenu, s'il est orné, c'est que ce monologue est d'abord lyrique, c'est-à-dire émanant de la plus intime émotion du 
personnage et s'efforçant au style sublime, en rapport avec le contenu de la méditation de Sénèque. Le personnage est prisonnier, et de cet état se libère et s'élève. De strophe en strophe, ce mouvement est répété : de la matière à l'âme, du monde sublunaire à la sphère céleste, de la tyrannie au repos. La sortie, l'envol s'achèvent par l'adresse à Dieu de la quatrième stance, qui est moins une apostrophe (rhétorique) qu'une prière. Il ne s'agit nullement dans ces stances d'un discours délibératif (à quoi on ramène parfois tout monologue dans la tragédie), mais d'une parole qui fait suite à la décision. Le sujet, placé devant sa disparition prochaine, donne l'essor à une parole au-delà du tragique, qu'on doit appeler philosophique, au sens montaignien du mot («Que philosopher c'est apprendre à mourir ») 5 .

Tel est en effet le thème central des stances de Sénèque : la mort consentie. Ce thème demande à être interprété. Au-delà de l'éthique individuelle dont il expose la force et la beauté, il rend perceptible la modélisation historique symbolisée par le conflit du sage et du tyran et par la mort du sage. Tristan met en scène de manière efficace la mutation qui affecte le statut de l'homme de pensée dans la première moitié du siècle : volé de son art par un pouvoir qui s'est emparé de l'éloquence et de la représentation persuasive; enfermé dans le lieu des discours corrompus; réfugié dans une parole sans effets sur le social et le politique. La mort acceptée rend manifeste ce retrait de la vie sociale ${ }^{6}$. Alors que la Renaissance avait connu une relation harmonieuse entre le Prince et le Sage, jusqu'aux figures présentant une parfaite synthèse, à la Pantagruel, l'âge baroque voit se briser cette complémentarité 7 . De la librairie de Montaigne à la salle d'eau où Sénèque entaille ses poignets amaigris, se raconte et se parachève l'histoire d'une séparation.

J'ai tenté de développer le paradoxe de cette mort consentie dans un essai récent ${ }^{8}$. La constitution du for intérieur comme liberté individuelle inaliénable contre les pressions de l'espace social me semblait avoir été acquise, au XVII siècle, en contrepartie d'une acceptation du jeu social et du jeu du pouvoir : intus ut libet, foris ut moris est. La figure qui guidait mon analyse était alors Pétrone, tel que le décrit Saint-Evremond, supprimant tout tragique et toute plainte dans son suicide, évitant sans apprêts tout pathos ${ }^{9}$. Un moment d'équilibre, marqué par la rencontre du libertinage philosophique et des théories de l'honnèteté, pouvait être ainsi dessiné, représenté par des hommes comme Méré, La Fontaine, Saint-Evremond

5. Le contenu des stances est inspiré par les œuvres mêmes de Sénèque, et notamment par les Lettres à Lucilius. V. La notice et les notes de l'édition citée.

6. Les traces ne manquent pas dans le texte, qui permettent de reconstruire un cadre objectif et évitent à ma lecture le reproche d'allégorisme. Ainsi l'accusation de «pédant hypocrite » portée par Sabine est une allusion précise à la dévalorisation des savoirs; la conversion de Sénèque suggérée à la fin (qui est sans doute un anachronisme) vient directement d'un ouvrage du Père Caussin, La Cour Sainte... De plus, d’autres textes du second quart du XVII ${ }^{\mathbf{c}}$ siècle, Faret, Sorel, Scarron, abordent le même problème.

7. L'étude historique est ici indispensable pour affiner l'approche. V. Notamment Roger Chartier, $L ' E ́ d u c a t i o n$ en France du $X V F$ au $X V I I I^{e}$ siècle, Soc. d’èd. d'enseign. sup., Paris, 1976.

8. L'Age libertin, chap. I, Minuit, 1987.

9. V. l'édition d'Alain Niderst, Saint-Evremond, Textes choisis, Éd. sociales, Paris, 1970 : «Jugement sur Sénèque, Plutarque et Pétrone $"$, pp. 63-71. Saint-Evremond est sévère sur Sénéque et en présente une image tout opposée à celle de Tristan: "J'estime le précepteur de Néron, l'amant d'Agrippine, l'ambiticux qui prétendait à l'empire. Du philosophe et de l'ècrivain, je ne fais pas grand cas : je ne suis touché ni de son stylc ni de ses sentiments. " V. aussi l'éd. des CEuvres complètes, par René Ternois. 
lui-même. La Mort de Sénèque, si elle croise la représentation pétronienne du retrait, en diffère pourtant. Elle ne répond pas au modèle épicurien de la sécession du sage et du jardin des délices, tels que le $X{ }{ }^{e}{ }^{e}$ siècle les a revisités, mais à un modèle stoïcien et chrétien de l'acceptation de la mort. Selon ce modèle le sage, s'il consent par son retrait à laisser le monde à son jeu, juge cependant et condamne ce jeu, et vise en s'en détachant à exercer sur lui une sorte d'action par défaut. Il faudra revenir sur ce point.

\section{Stances proches de la catastrophe}

Il s'agit maintenant, munis d'une problématique et, en somme, d'un paradigme, de poursuivre l'enquête sur les stances. Pour prendre au sérieux l'idée d'une forme littéraire, ou, plus exactement, d'un dispositif formel, et non simplement d'une métrique particulière, il faut écarter les stances contenues dans les pièces comiques et tragi-comiques; et, dans les tragédies, il ne faut retenir que les stances proches de la catastrophe, à l'acte $\mathrm{V}$ ou à l'acte IV. On ne tiendra donc pas compte, du moins à cette étape de la recherche, des stances délibératives, monologues ornés dont les plus célèbres se trouvent dans Le Cid (acte I), dans Andromède (acte III) ou dans Edipe (acte III)... Si l'on perd ainsi en extension, écartant nombre de stances, on gagne en compréhension, affinant et approfondissant le « noyau conceptuel ». Liant une métrique particulière à un sens et à une fonction spécifiques et fondamentaux, on cesse de la considérer comme un ornement pour la resémantiser : ce qui constitue une opération essentielle de toute véritable littérature. Cette resémantisation a affaire à l'histoire et au contexte historique d'autant plus évidemment que les stances ainsi délimitées se rencontrent essentiellement (et peut-être exclusivement) entre 1634 et 1646, dans le moment où cette pratique poétique est à son apogée.

C'est dans la Médée de Corneille, dit Lancaster, qu'apparaissent pour la première fois des stances dans la tragédie française ${ }^{10}$. Elles sont prononcées par Égée dans sa prison, en IV, V. On y trouve en germe les éléments que La Mort de Sénèque développe. Le héros y est seul, enfermé, et son discours constitue le monologue lyrique et sublime des stances devant la mort. Sa parole s'élève hors de sa prison pour s'adresser au Destin et au Dieu de l'amour; dans son ample mouvement, son apostrophe jouxte l'hypotypose, lorsque Égée anime les murs de son cachot '". Le personnage accepte sa disparition et s'y prépare. Pourtant, Égée ne joue qu'un rôle secondaire dans la pièce et sera délivré à la scène suivante. Il n'est ni un personnage tragique, ni une figure de sage, mais un roi qui a perdu la sagesse et la retrouve précisément dans la méditation des stances. Corneille propose une forme-thème, - et ce sont des pièces ultérieures qui vont en interpréter les virtualités.

10. Citć par J. Scherer, op. cil.

11. Marc Fumaroli a montré tout le parti que tire Corneille de cette figure dans $L$ 'Illusion comique, pièce qui succède à Médée. V. "Rhètorique et dramaturgie dans $L$ 'Illusion comique ". XVIF siècle, 1968, $\mathrm{n}^{\circ} 80-81$, pp. 107-132. 
En 1636, la Marianne de Tristan accorde une place décisive aux stances devant la mort. La pièce mériterait une analyse fouillée ; je me contenterai d'en mettre en évidence les aspects qui importent à la compréhension des stances.

Hérode, roi de Judée, a épousé Marianne, fille des princes légitimes dont il a éteint la lignée par l'assassinat. Il éprouve un amour passionné pour sa femme, qui se refuse à lui et le hait, poursuivie par le souvenir de ses meurtres. Hérode, tourmenté par la jalousie, prête l'oreille aux délateurs qui accusent Marianne de vouloir empoisonner le roi; il imagine de plus que sa femme le trompe avec son intendant. Il l'enferme, la fait juger sommairement et exécuter. Les stances trouvent place à l'acte IV, scène II, au moment où Marianne, dans sa prison, médite sur sa mort prochaine. C'est bien la situation des stances proches de la catastrophe: l'héroïne, solitaire, s'adresse à la puissance suprême dans un chant apaisé, au style sublime, en une parole qui s'élève au-dessus des contingences du corps et du monde, qui n'a plus contre la tyrannie d'aigreur ni de révolte mais l'accepte comme un mal inévitable. La condamnation à mort est ainsi transformée en une délivrance. Il faut citer intégralement ces stances, qui comptent parmi les beaux exemples lyriques dans la tragédie française :

Pour augmenter l'affront que l'injuste licence

A fait à l'innocence,

Un absolu pouvoir rend mon corps prisonnier :

Mais en quelque péril que le malheur m'engage,

J'aurai cet avantage

Que mon cœur pour le moins se rendra le dernier.

Ce jour s'en va borner la longueur de ma vie.

Je vois bien que l'envie

Travaille puissamment à creuser mon tombeau,

Et que la cruauté du Tyran qui m'opprime

Ne me suppose un crime

Que pour avoir sujet d'en commettre un nouveau.

Qu'il en use à son gré, me voilà toute prête

De payer de ma tête,

Afin de contenter ce cour dénaturé ;

Quelque horreur qu'en la mort on puisse reconnaître

Elle n'a qu'à paraître,

J'irai la recevoir d'un visage assuré.

Il est temps désormais que le Ciel me sépare

D'avecque ce barbare,

Son humeur et la mienne ont trop peu de rapport ;

La vertu respirant parmi l'odeur du vice

Eprouve le supplice

Du vivant bouche à bouche attaché contre un mort.

Auteur de l'Univers, souveraine puissance,

Qui depuis ma naissance

M'as toujours envoyé des matières de pleurs,

Mon âme n'a recours qu'à tes bontés divines.

Au milieu des épines,

Seigneur, fais-moi bientôt marcher dessus des fleurs. 
L'envol et l'élévation, qui marquent si puissamment les stances de Sénèque, ne forment pas ici le mouvement central. Ils ne sont perceptibles que dans l'apostrophe de la dernière strophe et dans le recours, topique, à l'héroïsme du personnage tragique. C'est ici un autre élément fondamental de la poétique baroque qu'il convient de relever ${ }^{12}$. La pièce est tout entière structurée sur le fait qu'Hérode et Marianne sont des contraires dont l'association forcée crée une permanente tension : la légitimité et l'usurpation, la vertu et le crime, la beauté et la laideur, la froideur et le désir (la glace et le feu, dit le texte)... C'est sur ces oppositions que repose le lyrisme des stances, nommées, détaillées, mais en même temps levées, jusqu'à la terrible antithèse qui fait la chute des quatre premières stances, avant l'appel à Dieu : «Du vivant bouche à bouche attaché contre un mort."

Ce jeu rhétorique et dramatique des antithèses est dénoué dans les stances, la séparation appelée par Marianne est effectuée par l'acceptation de la mort. Mais il faut aller plus loin. En consentant à mourir, Marianne donne son assentiment au contraire : à la mort certes, dont elle se fait une alliée, mais surtout à Hérode, cédant à sa folie et à sa tyrannie. L'assentiment n'est donc pas un ralliement, mais forme une nouvelle figure antithétique, à un niveau supérieur, un consentement qui reste antagoniste : Marianne laisse à Hérode sa place, en se servant de cet abandon pour s'en détacher, en utilisant cette défaite pour obtenir une victoire. On a là, transposée sur le plan dramaturgique où elle ouvre à la résolution, une forme de pensée souvent présente dans les figures antithétiques ou oxymoriques de la pointe maniériste ou baroque, comme une subtilité supplémentaire de l'esprit, qui le rend en même temps plus évanescent et plus fort. Ce mouvement complexe montre bien la différence des stances devant la mort d'avec les monologues tragiques "classiques ", qui sont des délibérations aveuglées (qu'on pense à Hermione ou à Phèdre), où les oppositions restent mécaniques et stériles.

\section{L'efficacité du martyre}

Relèvent, peu ou prou, d'une même analyse les stances prononcées par Mithridate, dans La Mort de Mithridate de La Calprenède (1636) :

Ceux qui font un bien véritable

D'un bonheur instable et mouvant,

Charmés d'un appât décevant,

Ne sont fondés que sur le sable.

$\ldots{ }^{13}$

Le roi tient une coupe pleine de poison, ses proches se sont éloignés, tous l'un après l'autre vont avaler le breuvage et s'effondrer sur scène, au long d'un acte interminable... La famille royale a décidé de se donner la mort pour laisser le champ libre à Pharnace, le fils vainqueur qui a pactisé avec les Romains. Ces stances contiennent les éléments qui nous apparaissent maintenant non seulement comme un dispositif

12. V. Jean Rousset, La Littérature de l'âge baroque en France, Paris, 1972.

13. Toujours dans le vol. II, op. cit., p. 189 ; ce sont les premiers vers des stances en V, I. 
récurrent, mais aussi comme une topique. On pourrait évoquer d'autres tragédies encore (tel l'Héraclius de Corneille, où les stances apparaissent en V, I) ; y intégrer certaines tragi-comédies dont les stances répondent à notre analyse (par exemple L'Amour tyrannique, de Scudéry, stances en V, I) ; examiner les comédies qui parodient ou renversent le thème en faisant de l'acceptation de la mort une découverte de l'amour: ainsi La Belle Alphrède de Rotrou (stances en IV, II ${ }^{14}$ ) ou Les Visionnaires de Desmarets de Saint-Sorlin, où un poète fait marché de stances macaroniques avec un riche amoureux ${ }^{15}$. On verrait s'établir des parentés, mais on perdrait l'acuité du thème central. En revanche, pour compléter l'enquête en la différenciant, il est nécessaire d'analyser deux pièces : Polyeucte, de Corneille (1643) et Saint-Genest de Rotrou (1646).

Polyeucte renverse les dieux païens, provoque les maitres romains, refuse les compromis suggérés par l'amour et l'honneur. Animé du zèle du martyr, il demande la mort. Le désir d'élever son âme, l'aspiration au salut ne composent qu'une part de cette ardeur à mourir. Il y en a une autre, que Corneille met en lumière avec une extrême finesse dans les détours de l'intrigue amoureuse dont Pauline est le centre. Polyeucte brûle de laisser la place à l'autre : à Sévère, son lit et sa femme; aux Romains, le gouvernement de son pays, dont il est l'hérititer légitime. Tel est chez lui l'assentiment au contraire, que sa dévotion excessive et emportée vient revêtir d'une parure sacrificielle. Les stances qu'il récite à l'acte IV, scène II, dans la prison, avant l'entrevue avec Pauline où il consommera leur rupture en lui faisant l'injure de la donner à Sévère, contiennent toute la complexité de ces mouvements. Polyeucte y renonce au monde et à ses plaisirs, mais aussi, au nom du Dieu vengeur, il prophétise la ruine prochaine des Romains, puis imagine l'avenir de ses proches après sa disparition. La sagesse du philosophe est remplacée par le zèle religieux, et le grand lyrisme cornélien, avec ses emportements et ses maniérismes, se donne libre cours :

Source délicieuse, en misères féconde ;

Que voulez-vous de moi, flatteuses voluptés?

Honteux attachements de la chair et du Monde,

Que ne me quittez-vous, quand je vous ai quittés?

Allez, honneurs, plaisirs, qui me livrez la guerre :

Toute votre félicité,

Sujette à l'instabilité,

En moins de rien tombe par terre;

Et, comme elle a l'éclat du verre,

Elle en a la fragilité.

14. Op. cit., vol. I, p. 837 , v. $1272-4$ :

Que la mort dont je meurs vaut bien mieux que la vie, Et qu'avec peu d'envic

Parmi de si beaux fers je vois la liberté !

15. Op. cit., vol. II, p. 448 , v. $925-31$ :

Toutefois, parmi ces ardeurs,

Tes hétéroclites froideurs

Causent une antipéristase ;

Ainsi mourant, ne mourant pas

Je me sens ravir en extase

Entre la vie et le trépas. 
$[\ldots]$

Saintes douceurs du Ciel, adorables idées,

Vous remplissez un cœur qui vous peut recevoir :

De vos sacrés attraits les âmes possédées

$\mathrm{Ne}$ conçoivent plus rien qui les puissent émouvoir.

Vous promettez beaucoup, et donnez davantage :

Vos biens ne sont point inconstants;

Et l'heureux trépas que j'attends

Ne vous sert que d'un doux passage

Pour nous introduire au partage

Qui nous rend à jamais contents 16

La pointe qui termine chaque stance, qui en fait la chute, témoigne de la virtuosité poétique de l'auteur jusqu'à la matérialisation graphique, puisque les cinq octosyllabes composent une manière de calligramme. Dans les strophes 2 et 3 , cette finale acérée est encore plus concrète, quand Polyeucte évoque le glaive et la foudre que Dieu tient prêts pour punir les Romains. La pointe se fait métaphore de l'action propre au martyr. Sa complexe synthèse des contraires, analysée précédemment sous l'angle de l'assentiment, doit l'être ici dans la perspective de l'action par défaut. Laisser sa place à l'autre, c'est aussi faire éclater sa position d'usurpateur et rendre irréparable l'injustice dont il est coupable. En les obligeant à le tuer, Polyeucte instille chez ceux qui restent le poison de la faute. Par le retrait infini de sa mort, le martyr crée un vide qui va aspirer ses bourreaux : c'est à ce mécanisme que répond l'invraisemblable cascade de conversions qui termine la pièce. Au-delà du «merveilleux chrétien ", dont la naïveté de vignette ne peut pas tromper longtemps, ce langage dévot soudainement apparu dans la bouche de ceux qui viennent de tuer Polyeucte illustre l'efficacité de cette action par défaut qu'on pouvait pressentir dans le retrait du sage. La mort de Marianne ni celle de Sénèque ne tiennent rien du prosélytisme, et pourtant elles aussi produisent des effets : Hérode devient fou, il maudit les Juifs et leur prédit des siècles de malheur; Néron, saisi d'une sombre mélancolie, pressent son délire criminel et sa fin prochaine. Pourtant, dans la tragédie du martyre, cette figure de l'absence irradiante, formulation chrétienne de la synthèse baroque des contraires, dénature quelque peu le thème stoïcien. La parole des stances cesse d'être celle d'un sujet qui s'affranchit des contraintes du monde. Elle n'est plus ce composé de lyrisme et de philosophie, ce chant argumenté du sage devant la mort ; elle devient le lieu d'un combat spirituel où la violence terrestre est remplacée par l'ardeur de mourir.

Les stances de Genest, dans la pièce de Rotrou (V, I), mettent l'accent sur cette transformation, en montrant un comédien devenu un guerrier. On reconnaît, dans ces stances, toutes les valeurs attachées au dispositif formel que nous avons décrit. Genest est seul, enchaîné dans un cachot, et son monologue n'a rien d'une délibération :

Par quelle divine aventure,

Sensible et sainte volupté,

16. Cité d'après d’èd. de Georges Couton, Pléiade, O.C., t. I, 1980, pp. 1026-1027. Il s'agit ici des première et dernière strophes. 


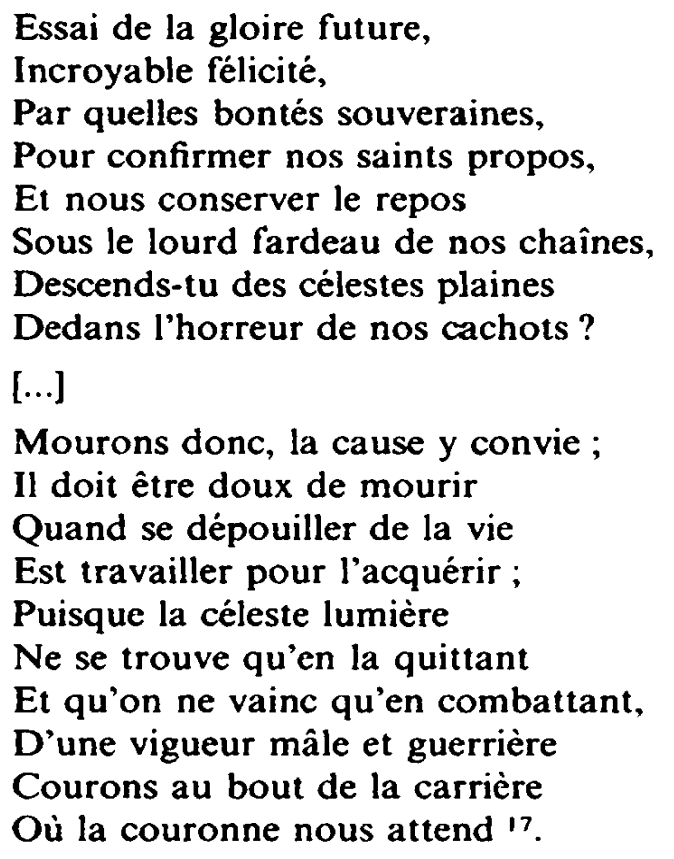

On est loin, dans cette précipitation vers la mort, de la sereine acceptation du sage. Si les stances continuent de magnifier une parole d'élévation et d'assentiment, elles procèdent d'une tout autre interprétation du mouvement libératoire que celle où atteignait Sénèque. Pourtant, Genest est lui aussi un homme de pensée; acteur et directeur de troupe écouté, son aventure est celle d'une découverte de la parole vraie ${ }^{18}$. Loin de condamner le théâtre, cette découverte confirme avec éclat les pouvoirs de la représentation: l'effet du représenté change le représentant. Le théàtre, divertissement profane, peut devenir le véhicule d'une parole sacrée, tout comme les stances, qui réunissent la plus grande intensité ornementale, et disent le plus total dépouillement.

\section{Anthropologie et histoire}

On pourrait conclure dans la perspective d'une anthropologie historique inspirée de Dumézil. Entre les bellatores et les oratores. une crise s'est déclarée. Les uns prétendent s'emparer d'une fonction essentielle des autres ; ceux-ci, à leur tour, s'inspirent des comportements guerriers dans des buts spirituels. Les compétences et les prérogatives traditionnellement acceptées demandent à être révisées. Les stances devant la mort sont au cœur de ce débat : elles montrent que les détenteurs du savoir, de la pensée et de la parole sont menacés par ceux qui devraient les protéger, que le savoir et la parole sont asservis au pouvoir, - sauf à recourir à un principe qui transcende la division des ordres elle-même.

17. Théâtre du XVII siècle, t. I, pp. 994-995, première et dernic̀re strophes.

18 .

Et qu'il a bien voulu. par son impiété,

D'unc feinte, en mourant, faire une vérité.

Ce sont les derniers mots de la pièce. Sur le Saint-Genest, v. Jean Rousset, L'Intérieur et lextérieur, Paris, 1976: Georges Forestier, Le Théâtre dans le théâtre sur la scène française du XVIF siècle, Droz, Genève, 1981 . 
Mais il faut surtout compléter cette esquisse par des considérations historiques. Le dispositif formel et thématique qu'on a analysé est précisément daté : là réside un de ses intérêts majeurs. On l'a dit, les premières stances tragiques apparaissent en 1634 ; trente ans plus tard, elles ont disparu. Dans La Thébaïde, sa première pièce (1664), Racine place des stances en V, I, dans la bouche d'Antigone. Elles sont un pur monologue délibératif : la parole lyrique et philosophique est définalisée, le dispositif est mésinterprété. Racine n'a que faire des stances, que d'ailleurs le public et les théoriciens n'apprécient plus. Durant la brève période de leur essor, on peut distinguer deux époques. Aux alentours de 1635, les héros «à stances » sont des rois et des reines, personnel convenu de l'héroïsme et de la tragédie (Égée, Marianne, Mithridate). Les stances disent leur acceptation du malheur et leur libération; mais l'élément philosophique en elles n'est pas dégagé, comme si les virtualités du dispositif restaient inactivées. Dix ans plus tard, les héros qui disent des stances prennent un visage singulier : un converti, un philosophe, un comédien. Les stances alors recontextualisent le dispositif, l'adaptent à la situation des personnages et en font un outil interprétatif capable de faire apparaître symboliquement le statut des «clercs » devant le pouvoir. La pensée libre, tout comme la foi sans compromission, n'ont pas leur place dans l'espace politique, dit cette réinterprétation, donnant une représentation d'une réalité socio-culturelle qui parcourt tout le siècle sous divers visages : le clientélisme outré pratiqué par les grands, l'asservissement des écrivains à l'éloge du roi, la dépréciation des connaissances spécialisées dans les milieux mondains, le nécessaire travertissement de toute pensée critique, mais aussi bien la mise à l'écart de toute profession intransigeante... On aurait tort de vouloir ramener à l'unité des phénomènes si divers, sinon par la médiation de ce « noyau conceptuel » qui permet de les penser ensemble, d'en saisir l'enjeu avant de rendre justice à leurs différences. Mais on aurait tort aussi bien de ne pas voir qu'une même modélisation symbolique, venue d'une mutation historique profonde, gouverne ces manifestations divergentes.

Dans $L e P l i{ }^{19}$, Gilles Deleuze compare la monade leibnizienne à une chapelle baroque. On pourrait développer cette comparaison pour la stance également. Lieu du retrait ct de l'intime à l'intérieur d'un plus vaste monument, mais aussi lieu de l'éclairement par le haut, de l'aspiration vers la lumière d'en-haut, de l'envol de l'âme et de la parole vers leur principe ; lieu hyperboliquement clos sur ses symétries, et lieu d'éclatement lyrique de la matière et des sens spiritualisés. Dans la chapelle baroque, les contraires travaillent à l'unité du tout. Comme elle, la stance est le lieu - verbal et dramatique - du dépouillement et de l'excès, de la clôture et de l'échappement, où le pathos se convertit en ethos. L'étymologie même (stanze, de stare : repos et clôture) et le détail formel sont alors rendus à la vie, revisités par le sens dans une compréhension qui fait de l'ornementation et de la disposition dramatique une reprise symbolique d'un contenu qui s'étend, par ondes, jusqu'à comprendre le temps de son apparition et les questions, multiples et parfois confuses, que les hommes s'y sont posées.

19. Ed. de Minuit, 1988. 